\title{
Including UPFC Dynamic Phasor Model into Transient Stability Program
}

\author{
Haoming Liu, Haojun Zhu, Yang Li, and Yixin Ni, Senior Member, IEEE
}

\begin{abstract}
In this paper a novel time simulation approach is introduced to implement transient stability analysis with FACTS devices, in which FACTS devices will use dynamic phasor models and interface properly with conventional electromechanical transient-model-based stability program. The unified power flow controller (UPFC) is used as an example to demo the realization of the approach. In the paper, the UPFC dynamic phasor model and control scheme are presented first and followed by the interface formulation. Sequential solution method is proposed to implement step by step simulation. Computer test results from a SMIB power system show clearly the accuracy of dynamic phasor model as compared with EMT model for UPFC; while computer test results from a two-area power system illustrate solidly the effectiveness of the suggested interface and simulation approach to include dynamic-phasor-based FACTS device models into conventional transient stability program.
\end{abstract}

Index Terms--Hybrid simulation, dynamic phasor, flexible ac transmission system (FACTS), unified power flow controller (UPFC), transient stability.

\section{INTRODUCTION}

$\mathrm{F}_{\mathrm{s}}$ ACTS devices play an important role in modern largescale interconnected power systems. They can be used not only for increasing system operation flexibility and controllability, but also for enhancing system stability, hence the better utilization of existing power systems can be realized.

However, FACTS devices are based on switching circuits composed of power electronic elements such as thyristors, GTOs, MOSFETs, and diodes. It is difficult to analyze the dynamics of entire system with FACTS devices, because accurate modeling of power electronic devices needs electromagnetic transient (EMT) models which is timeconsuming in simulation for the tiny step length used. In the conventional power system stability programs, electrome-

This project was jointly supported by the National Key Basic Research Special Fund of China (2004CB217900), the National Natural Science Foundation of China (50337010), the National Natural Science Foundation of China (60474078) and the Research Grant Council of Hong Kong SAR Government (CityU 1/00C).

Haoming Liu and Yang Li are with Department of Electrical Engineering, Southeast University, Nanjing, 210096, China. (e-mail: hmliu@eee.hku.hk, li_yang@seu.edu.cn )

Haojun Zhu is with the College of Electrical Engineering, South China University of Technology, Guangzhou, 510640, China. (e-mail: zhuhaojun@sina.com )

Yixin Ni is with the Department of Electrical and Electronic Engineering, the University of Hong Kong, Hong Kong. (e-mail: yxni@eee.hku.hk ) chanical transient model is widely used. How to fill in the gap between the two types of models and make accurate and efficient simulation is an important issue in modern power system stability analysis with power electronic devices. It is clear that in order to study the impacts of FACTS devices on power system dynamic performance, there is an increasing need for simplified models of FACTS devices with sufficient engineering accuracy which will allow fast and accurate modeling, simulation and control design for overall system stability purpose.

In order to describe the dynamic behavior of the switching FACTS devices correctly, a precise approach is provided by sampled-data models [1]. By sampling the full system states per switching cycle, a nonlinear sampled-data model could be established, which can predict system behavior very well. However, it can become cumbersome and impractical for large power systems, and the model derivation is relatively complicated. In this paper, dynamic phasor modeling of power electronic devices is suggested, which is based on timevarying Fourier coefficient series of the system variables [2], [3]. By keeping the dominant components in Fourier coefficient series, we can catch the significant impacts of the power electronic devices on system stability. Dynamic phasor theory has great potential to be used in power system stability analysis for FACTS device modeling and fill in the gap between EMT model and quasi-steady state models.

The unified power flow controller (UPFC) is a member of the FACTS family with very attractive features. During the past decade, many researches on the principles and simulation of UPFC have been made. In digital simulation, electromagnetic transient programs are widely used for UPFC analysis [4]. However in the study of power system transient and steady-state stability, a dynamic model for UPFC is required to interface UPFC to the quasi-static model of ac transmission network and to analyze its effects on large-scale power systems. Some papers suggest UPFC power frequency models which were limited to be used in one machine to infinite bus system [5], [6]. Some other papers developed UPFC power frequency models with its de link capacitor dynamics neglected or with some other approximations that might lead to undesirable error [7], [8].

In this paper, the UPFC dynamic phasor model is derived with its de link capacitor dynamics included. A novel UPFCnetwork interface is suggested for hybrid model simulation with FACTS devices in dynamic phasor model and the ac 
system in conventional electromechanical transient model. In this model, the UPFC shunt element control is to keep the UPFC terminal ac bus voltage and the dc link capacitor voltage constant, and the series element control is to keep constant the active and reactive power of the line. The UPFC dynamic phasor model has been incorporated into the conventional power system transient programs with the proposed UPFC-network interface. Its application is illustrated with a SIMB system and a 2-area interconnected power system [9]. The simulation results show that the suggested UPFC dynamic phasor model have good accuracy and the UPFC-network interface method work effectively in the study of overall system dynamic behavior.

\section{DYNAMIC PHASOR MODEL OF UPFC}

\section{A. Outline of the dynamic phasor}

The method of dynamic phasor is based on the timevarying Fourier coefficients [2]. A possibly complex timedomain waveform $x(\tau)$ can be represented on the interval $\tau \in(t-T, t]$ using a Fourier series of the form

$$
x(\tau)=\sum_{k=-\infty}^{\infty} X_{k}(t) e^{j k \omega_{s} \tau}
$$

where $\omega_{s}=2 \pi / T$ and $X_{k}(t)$ are the Fourier coefficients and named here as dynamic phasor. The $k$ th phasor at time $t$ can be determined by the following expression:

$$
X_{k}(t)=\frac{1}{T} \int_{t-T}^{t} x(\tau) e^{-j k \omega_{s} \tau} d \tau=\langle x\rangle_{k}(t)
$$

The phasor $\langle x\rangle_{k}(t)$ here are all complex quantities, which satisfy the following:

$$
\langle x\rangle_{k}=\langle x\rangle_{k}^{r}+j\langle x\rangle_{k}^{i}=\langle x\rangle_{-k}^{*}=\left(\langle x\rangle_{-k}^{r}+j\langle x\rangle_{-k}^{i}\right)^{*}
$$

where the superscripts $r$ and $i$ denote the real and imaginary parts of the defined quantities respectively, and "*” denotes the complex conjugation.

There are two key and useful properties of the dynamic phasor:

Differentiation of dynamic phasor: For the $k$ th Fourier coefficient, the differential with time satisfy the following formula:

$$
\frac{d\langle x\rangle_{k}}{d t}(t)=\left\langle\frac{d x}{d t}\right\rangle_{k}(t)-j k \omega_{s}\langle x\rangle_{k}(t)
$$

Product of dynamic phasor: For two waveforms $x(t)$ and $q(t)$, the $k$ th phasor of their product can be obtained by:

$$
\langle x q\rangle_{k}=\sum_{i}\langle x\rangle_{k-i}\langle q\rangle_{i}
$$

Dynamic phasor method is based on the idea of frequency decomposition, and focus on the dynamics of the significant Fourier coefficient. By truncating unimportant higher order series and keep only those significant series, the dynamic phasor model can catch the dynamic behavior of the original detail model. A new state-space model can be obtained when we consider these reserved phasors as state variables. The model is simplified, and can keep the nonlinear of original model to large extent.

Dynamic phasor can be used to model the polyphase systems under unbalanced operation including electronic converters [3]. The emphasis of this paper is aimed at the analysis of three phase balanced situation.

\section{B. Modeling UPFC using dynamic phasor method}

A UPFC (see Fig.1) consists of two solid-state voltage source converters (VSC-E and VSC-B), which are connected through a common DC link capacitor. VSC-E realizes constant voltage control of $V_{S}$ and $V_{d}$; while VSC-B is used for series voltage $\dot{V}_{p q}$ control on both magnitude and phase angle which can in turn realize line power or series compensation etc. control strategy. The variables used in the paper are shown in Fig. 1, where $\left(\dot{V}_{E}, \dot{I}_{E}\right),\left(\dot{V}_{B}, \dot{I}_{B}\right)$ : ac terminal voltage and current of VSC-E and VSC-B respectively; $\left(m_{E}, \delta_{E}\right)$ and $\left(m_{B}, \delta_{B}\right)$ : modulation ratio of PWM control and firing angle of VSC-E and VSC-B respectively. And the relations of the converter dc- and acside voltages are (see Fig. 1)

$$
\left\{\begin{array}{l}
V_{E}=m_{E} V_{d} \\
V_{B}=m_{B} V_{d}
\end{array}\right.
$$

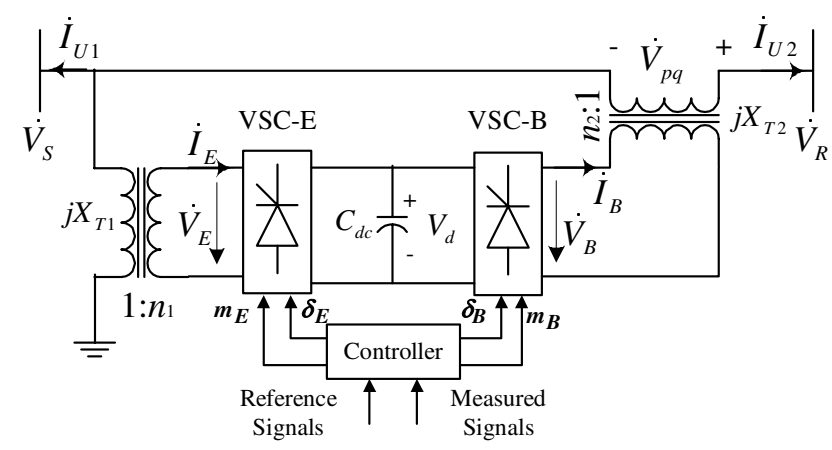

Fig. 1. Schematic diagram of UPFC

The phase angles of $\dot{V}_{E}$ and $\dot{V}_{B}$ are denoted as $\theta_{E}$ and $\theta_{B}$ which are controlled by the firing angle $\delta_{E}$ and $\delta_{B}$ of the two converters and have the relation $\left(\theta_{S}\right.$ : the phase angle of $\left.\dot{V}_{S}\right)$ as follows:

$$
\left\{\begin{array}{l}
\theta_{E}=\theta_{S}-\delta_{E} \\
\theta_{B}=\theta_{S}-\delta_{B}
\end{array}\right.
$$

For simplicity, we assume that the 3 phase ac system is symmetric; the converter valves are ideal; and converter transformer is lossless.

Taking phase a as the reference phase, Fig. 2 shows the equivalent circuit of phase a with its valve switching simulated by ideal switch-state functions $S_{E a}$ and $S_{E a}^{\prime}$ $\left(S_{E a}+S_{E a}^{\prime}=1\right)$. A small resistance $r_{s}$ is inserted to consider equivalent power loss. It is clear that $v_{E a}$ and $i_{E a}$ have the relation (' $n$ ' is the ac system neutral point) 


$$
\begin{aligned}
v_{E a} & =v_{E a H}+v_{H n} \\
& =\left[\left(i_{E a} r_{s}+v_{d c}\right) \cdot S_{E a}+\left(i_{E a} r_{s}\right) \cdot S_{E a}^{\prime}\right]+v_{H n}
\end{aligned}
$$

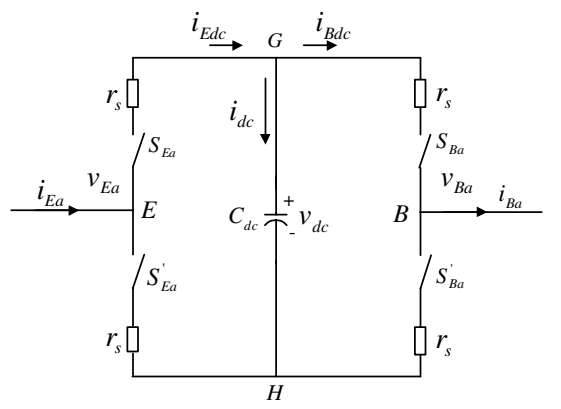

Fig. 2. Equivalent circuit of phase a of UPFC

For a balanced ac system, it is easy to derive

$$
v_{H n}=-\frac{1}{3} v_{d c} \sum_{j=a, b, c} S_{E j}
$$

Substituting (9) into (8), we have

$$
v_{E a}=i_{E a} r_{s}+v_{d c} \cdot S_{E a}-\frac{1}{3} v_{d c} \sum_{j=a, b, c} S_{E j}
$$

The dc capacitor dynamics can be described as

$$
C_{d c} \frac{d v_{d c}}{d t}=i_{d c}=i_{E d c}-i_{B d c}=\sum_{j=a, b, c}\left(i_{E j} S_{E j}-i_{B j} S_{B j}\right)
$$

According to (10) and (11), the dynamic phasors of $v_{E a}$ and $v_{d c}$ are,

$$
\begin{aligned}
& \left\langle V_{E a}\right\rangle_{1}=\left\langle I_{E a}\right\rangle_{1} r_{s}+\left\langle v_{d c}\right\rangle_{0} \cdot\left\langle S_{E a}\right\rangle_{1}-\frac{1}{3}\left\langle v_{d c}\right\rangle_{0} \sum_{j=a, b, c}\left\langle S_{E j}\right\rangle_{1} \\
& \frac{d\left\langle v_{d c}\right\rangle_{0}}{d t}=\frac{1}{C_{d c}} \sum_{j=a, b, c}\left(\left\langle i_{E j}\right\rangle_{1}\left\langle S_{E j}\right\rangle_{-1}-\left\langle i_{B j}\right\rangle_{1}\left\langle S_{B j}\right\rangle_{-1}\right)
\end{aligned}
$$

An equation similar to (12) can also be developed for series converter VSC-B. Still considering the balanced operation of three phases, we can simplify the three phase model to reference phase a model only.

The switch-state functions $S_{E a}, S_{E a}^{\prime}$ are determined by the PWM control, and they are discrete, periodic function of time. For stability study, we only filter out the fundamental wave component and dc component of the switching functions $S_{E a}$ [10], which can be expressed as

$$
d_{E j}=\frac{m_{E}}{2} \cos \left(\omega t-\delta_{E}-\sigma_{j}\right)+\frac{1}{2}
$$

where, $j=a, b, c$, and $\sigma_{a}=0, \sigma_{b}=\frac{2}{3} \pi, \sigma_{c}=\frac{4}{3} \pi$. For phase a, the dynamic phasors of $\mathrm{dc}$ and fundamental wave components are

$$
\left\langle d_{E a}\right\rangle_{0}=\frac{1}{2},\left\langle d_{E a}\right\rangle_{1}=\frac{m_{E}}{4} e^{j \theta_{E}},\left\langle d_{E a}\right\rangle_{-1}=\frac{m_{E}}{4} e^{-j \theta_{E}}
$$

Substituting dynamic phasor $d_{E j}$ in (15) for $S_{E j}$ in (12) and separating real and imaginary parts, we obtain the dynamic phasor model of UPFC:

$$
\left\{\begin{aligned}
\left\langle V_{E a}\right\rangle_{1}^{r} & =\left\langle I_{E a}\right\rangle_{1}^{r} \cdot r_{s}+\left\langle V_{d c}\right\rangle_{0} \cdot \frac{m_{E}}{4} \cos \theta_{E} \\
\left\langle V_{E a}\right\rangle_{1}^{i} & =\left\langle I_{E a}\right\rangle_{1}^{i} \cdot r_{s}+\left\langle V_{d c}\right\rangle_{0} \cdot \frac{m_{E}}{4} \sin \theta_{E} \\
\left\langle V_{B a}\right\rangle_{1}^{r} & =-\left\langle I_{B a}\right\rangle_{1}^{r} \cdot r_{s}+\left\langle V_{d c}\right\rangle_{0} \cdot \frac{m_{B}}{4} \cos \theta_{B} \\
\left\langle V_{B a}\right\rangle_{1}^{i} & =-\left\langle I_{B a}\right\rangle_{1}^{i} \cdot r_{s}+\left\langle V_{d c}\right\rangle_{0} \cdot \frac{m_{B}}{4} \sin \theta_{B} \\
\frac{d\left\langle V_{d c}\right\rangle_{0}}{d t} & =\frac{m_{E}}{C_{d c}} \frac{3}{2}\left[\left\langle I_{E a}\right\rangle_{1}^{r} \cdot \cos \theta_{E}+\left\langle I_{E a}\right\rangle_{1}^{i} \cdot \sin \theta_{E}\right] \\
& -\frac{m_{B}}{C_{d c}} \frac{3}{2}\left[\left\langle I_{B a}\right\rangle_{1}^{r} \cdot \cos \theta_{B}+\left\langle I_{B a}\right\rangle_{1}^{i} \cdot \sin \theta_{B}\right]
\end{aligned}\right.
$$

where, $\left\langle V_{d c}\right\rangle_{0}$ is a state variable; $m_{E}, m_{B}, \delta_{E}, \delta_{B}$ are control variables, which can be acquired from the control system of UPFC. Interfacing (16) with ac network equations, the overall system can be solved.

\section{UPFC main control}

The shunt and series VSC control strategies adopted in the paper are shown in Fig. 3 and Fig. 4 respectively.

In Fig. 3, the constant ac terminal bus voltage control is achieved by controlling $m_{E}$ of the PWM controller of VSC-E, and the constant dc link capacitor voltage control realized by controlling the firing angle $\delta_{E}$ of VSC-E. The controller is modeled as a simplified 1st order inertia block. There is no difficulty to consider complex control strategies.

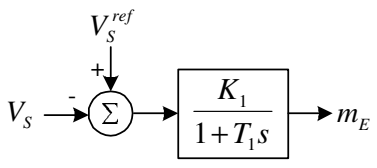

(a) The constant UPFC ac terminal bus voltage control Fig. 3. The UPFC shunt element control

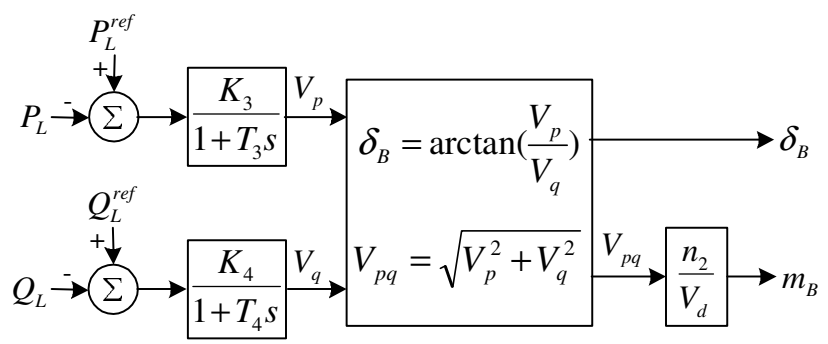

constant active and reactive power flow control

Fig. 4. The UPFC series element control

In Fig. 4, the constant real and reactive power flow control is achieved by controlling $m_{B}$ and $\delta_{B}$. It is clear that, the UPFC output series compensation voltage $\dot{V}_{p q}$ can be decomposed as $\dot{V}_{p}$ and $\dot{V}_{q}$. And the former is perpendicular to $\dot{V}_{S}$ and has strong impacts on real power flow, while the latter is in phase with $\dot{V}_{s}$ and has significant effects on reactive power flow. 
If the phase angle $\theta_{S}$ of $\dot{V}_{S}$ and UPFC control output $\left(m_{E}, \delta_{E}, m_{B}, \delta_{B}\right)$ are known, the dynamic phasor model of UPFC (see (16)) can be used to represent UPFC and interface to ac system for transient stability analysis.

\section{INTERFACE OF UPFC TO THE AC NETWORK}

In this paper, the system including UPFC should be divided into two parts, see Fig. 5. One is UPFC device, which should be modeled using dynamic phasor method, named as UPFCsubsystem. The other is the remainder parts include generators, loads, network and so on, which would be described using quasi-static model, called as ac networksubsystem.

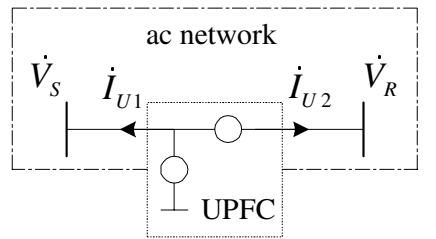

Fig. 5. The interface of UPFC with network

Due to different modeling methods are adopted to the two subsystems, they should be considered respectively, and the interaction should be analyzed. For UPFC-subsystem, the device could be equivalent as two voltage sources with inner inductance, $\dot{V}_{1}$ and $\dot{V}_{p q}$, coupling with network-subsystem (see Fig. 1). Considering the system is often described with equation $Y \dot{U}=\dot{I}$ in quasi-static model, the convenience and feasible interface variables are equivalent currents $\dot{I}_{U 1}$ and $\dot{I}_{U 2}$ injecting from UPFC-subsystem into network-subsystem. $\dot{V}_{1}$ and $\dot{V}_{p q}$ should be pre-decided to obtain the interface variables, while whose phase angles have the relation w.r.t. the phase angle of $\dot{V}_{S}$ (see (7)), and $\dot{V}_{S}$ is an undetermined variable. So the iteration is necessary to obtain the bus voltage $\dot{V}_{S}$ between the two subsystems.

In the interface calculation, we assume that the bus admittance matrix has been reduced to generator internal buses with UPFC ac terminal buses remained. The corresponding reduced bus admittance matrix equation takes the form:

$$
\left[\begin{array}{ll}
Y_{11} & Y_{12} \\
Y_{21} & Y_{22}
\end{array}\right]\left[\begin{array}{c}
\dot{V}_{G} \\
\dot{V}_{U}
\end{array}\right]=\left[\begin{array}{c}
\dot{I}_{G} \\
\dot{I}_{U}
\end{array}\right]
$$

here, $\dot{V}_{G}$ and $\dot{I}_{G}$ are generator internal bus voltage and injection current behind the subtransient reactance. $\dot{V}_{U}=\left[\begin{array}{ll}\dot{V}_{S} & \dot{V}_{R}\end{array}\right]^{T}$ are ac terminal bus voltages of the UPFC, and $\dot{I}_{U}=\left[\begin{array}{ll}\dot{I}_{U 1} & \dot{I}_{U 2}\end{array}\right]^{T}$ are injection currents to the network from UPFC.

Referring to Fig. 1, the UPFC currents injecting to the ac network can be expressed by

$$
\left\{\begin{array}{l}
\dot{I}_{U 1}=-\frac{\dot{V}_{S}-\dot{V}_{1} / n_{1}}{j X_{T 1}}-\dot{I}_{U 2} \\
\dot{I}_{U 2}=\frac{\dot{V}_{S}-\dot{V}_{R}+\dot{V}_{p q}}{j X_{T 2}}
\end{array}\right.
$$

If denote $Y_{22}=\left[\begin{array}{ll}Y_{S S} & Y_{S R} \\ Y_{R S} & Y_{R R}\end{array}\right], Y_{21} \dot{V}_{G}=\left[\begin{array}{l}\dot{I}_{G 1} \\ \dot{I}_{G 2}\end{array}\right]$, we can obtain the following formula,

$$
\begin{gathered}
Y_{22}^{\prime} \cdot \dot{V}_{U}=\dot{I}_{U}^{\prime} \\
\text { where, } Y_{22}^{\prime}=\left[\begin{array}{cc}
Y_{S S}+Y_{R S}+\frac{1}{j X_{T 1}} & Y_{S R}+Y_{R R} \\
Y_{R S}-\frac{1}{j X_{T 2}} & Y_{R R}+\frac{1}{j X_{T 2}}
\end{array}\right], \\
\dot{I}_{U}^{\prime}=\left[\begin{array}{c}
\frac{\dot{V}_{1}}{j n_{1} X_{T 1}}-\dot{I}_{G 1}-\dot{I}_{G 2} \\
\frac{\dot{V}_{p q}}{j X_{T 2}}-\dot{I}_{G 2}
\end{array}\right] .
\end{gathered}
$$

The iteration of UPFC-ac network are listed as follows:

Step 1: Determined the initial voltages of $\dot{V}_{S}^{(0)}, \dot{V}_{R}^{(0)}$;

Step 2: If $\dot{V}_{U}^{(k)}$ is known, calculate $\dot{I}_{U}^{\prime(k+1)}$, then solve (17) for $\dot{V}_{U}^{(k+1)}$;

Step 3: If the difference of $\dot{V}_{U}^{(k+1)}$ and $\dot{V}_{U}^{(k)}$ is less than the given tolerance, then $\dot{V}_{U}^{(k+1)}$ is considered as the final solution. Otherwise go to step 4;

Step 4: Update $\dot{V}_{U}^{(k)}$ by $\dot{V}_{U}^{(k+1)}$, and repeat steps 2 and 3 till convergence is reached.

\section{COMPUTER TEST RESUlTS}

\section{A. SMIB system}

In order to verify the accuracy of the dynamic phasor model, we select a SIMB system, and compare the simulation results of the dynamic phasor model with those of EMT model using MATLAB/SIMULINK toolbox.

In this case, at 0.1 second, modulation ratio $m_{B}$ in series side changes from 0.4 to 0.7 . The simulation results for UPFC dc capacitor voltage and ac line current are shown in Fig. 6.

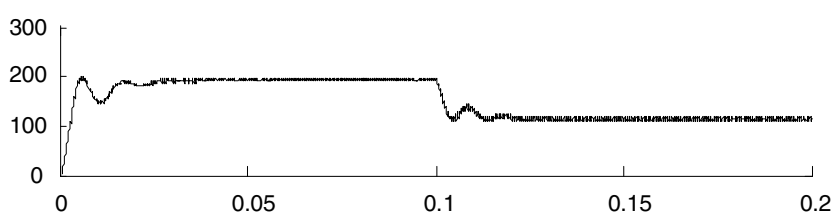

(a) dc capacitor voltage using EMT model (V/s)

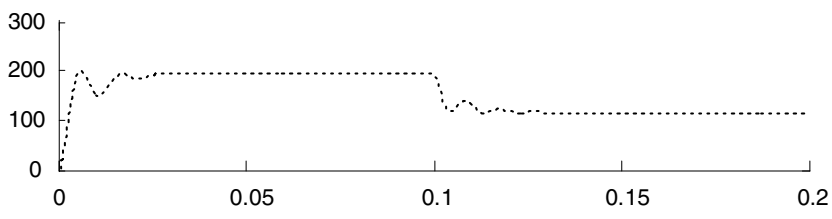

(b) dc capacitor voltage using DP model (V/s) 


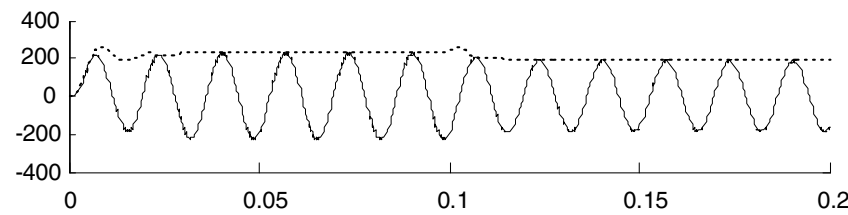

(c) ac line current of phase a in series side $(\mathrm{A} / \mathrm{s})$ - electromagnetic transient (EMT) …...dynamic phasor (DP) Fig. 6. Comparison of the two models

Fig. 6 (a) and (b) show that the de voltage based on EMT model is almost the same as the dc voltage based on dynamic phasor, and Fig. 6 (c) shows that the waveform of phase a current in series side using dynamic phasor could envelop the corresponding waveform using EMT simulation. The test illustrates that simulation results using dynamic phasor model have satisfied accuracy as compared with EMT model.

\section{B. 2-area interconnected system}

A 4-generator 2-area interconnected power system [9] is used for the computer test (see Fig. 7). In this case, the subtransient model is used for the generators with simplified third-order excitation control. The mechanical power of each generator is taken as a constant. Loads are expressed as constant impedance and the ac network is linear. All the parameters of UPFC and the parameters of UPFC main control are the same as [11].

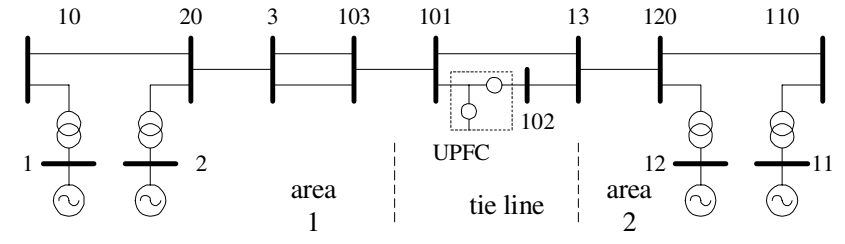

Fig. 7. 4-generator 2-area test system

The disturbance used is a three-phase earth fault on bus 3 at 0.5 second, and disappear in 0.1 second. Three cases are studied. They are: (i) the system has no UPFC; (ii) the system has UPFC and uses traditional phasor (TP) model for UPFC; (iii) the system has UPFC and uses dynamic phasor (DP) model for UPFC. The results are shown in Fig. 8-10 respectively. Each figure includes (a) voltage magnitude of bus 101, (b) active flow of the controlled line 102-13, (c) reactive flow of the controlled line.
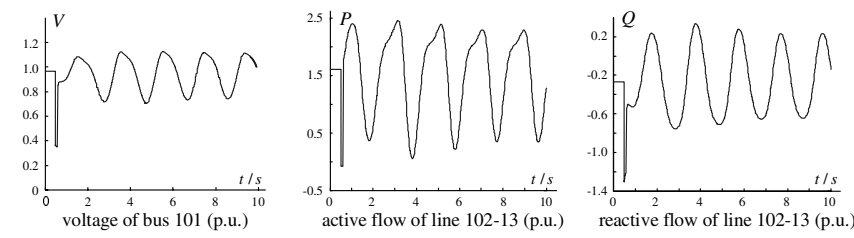

Fig. 8. The results of the system without UPFC
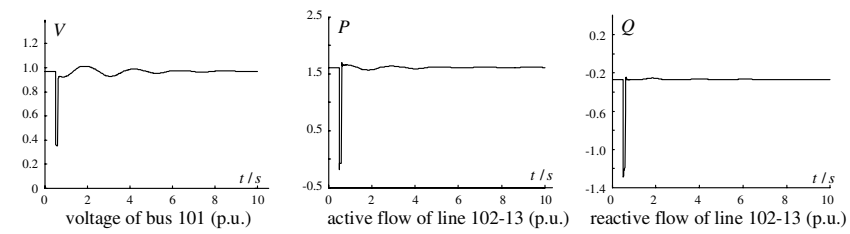

Fig. 9. The results of the system with UPFC using TP
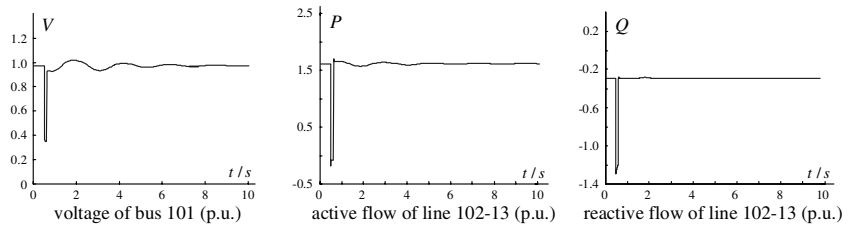

Fig. 10. The results of the system with UPFC using DP

The following observations can be made:

1) The results of the systems with UPFC using TP and DP models are consistent.

2) The voltage of bus 101 in the three figures show that the constant UPFC terminal bus voltage control is necessary to maintain the voltage stability after disturbance.

3) After the first swing, the constant power flow control can recover the controlled line power flow to the setting point very quickly, which is extremely desirable.

4) The time simulation shows very good convergence in UPFC-ac network interface; in the meantime, using dynamic phasor model for UPFC has marginal CPU time increase, which proves that the suggested UPFC model and interface method work very well in transient stability analysis.

\section{CONCLUSION}

This paper introduced a novel hybrid simulation method for the power system with FACTS devices. A dynamic model of UPFC are developed. The test results show that the suggested UPFC dynamic phasor model and the proposed UPFC-ac network interface algorithm could work very well in the study of power system dynamics with satisfactory convergence and accuracy.

\section{REFERENCES}

[1] X. D. Liu, Y. Yang, and C. Chen, "SSR computational analysis in TCSC system based on sample-data model," Proceedings of the CSEE, vol. 21, pp. 1-5, Feb. 2001.

[2] S. R. Sanders, J. M. Noworolski, X. Z. Liu, and G. C. Verghese, "Generalized averaging method for power conversion circuits," IEEE Trans. Power Electronics, vol. 6, pp. 251-259, Apr. 1991.

[3] A. M. Stankovic and T. Aydin, "Analysis of asymmetrical faults in power systems using dynamic phasors," IEEE Trans. power systems, vol. 15, pp. 1062-1068, .2000.

[4] M. P. Bottino, B. Delfino, and G. B.. Denegri, "FACTS for more effective networks: unified power flow controller using PSCAD/ EMTDC code," In EPRI - the future of power delivery conference, Washington, DC, 1996.

[5] R. Mihalic, P. Zunko, and D. Povh. "Improvement of transient stability using unified power flow controller," IEEE Trans. Power Delivery, vol. 11, pp. 485-492, Jan. 1996.

[6] K. S. Smith, L. Ran, and J. Penman, "Dynamic modeling of a unified power flow controller," IEE Proc. Gener. Transm. Distrib., vol. 144, pp.7-12, Jan. 1997.

[7] M. Noroozian, L. Angguist, M. Ghandhari, and G. Andersson, "Improving power system dynamics by series-connected FACTS devices," IEEE Trans. Power Delivery, vol. 12, pp. 1635-1641, Oct. 1997.

[8] S. Limyingcharoen, U. D. Annakkage, and N.C. Pahalawaththa, "Effects of unified power flow controllers on transient stability," IEE Proc. Gener. Transm. Distrib., vol.145, pp. 182-188, Mar. 1998. 
[9] P. Kundur, Power system stability and control, vol. I. New York: MacGraw-Hill, 1994, pp. 813-816.

[10] N. Nabavi and M. E. Iravani, "Steady-state and dynamic models of unified power flow controllers (UPFC) for power system studies," IEEE Trans. Power Systems, vol. 11, pp. 1937-1943, Nov. 1996.

[11] Z. Y. Huang, Y. X. Ni, C. M. Shen, F. F. Wu, S. S. Chen, and B. L. Zhang, "Application of unified power flow controller in interconnected power systems - modeling, interface, control strategy, and case study," IEEE Trans. Power Systems, vol. 15, pp. 817-824, Nov. 2000.

\section{BIOGRAPHIES}

Haoming Liu was born in Jiangsu, China, on Feb, 1977. He received his B. Eng., M. Eng., and Dr. Eng. from Nanjing University of Science and Technology, China in 1998, 2001 and 2003 respectively. His research interests are power system stability and control and electricity markets. Currently, he is a postdoctor at the department of Electrical Engineering, Southeast University, China.

Haojun Zhu was born in Guangdong, China, on Feb, 1978. He received his B.S. degree, M.S. degree from South China University of Technology,China in 2001 and 2003 respectively. He is currently a graduate student for Ph.D. degree in the College of Electrical Engineering of the South China University of Technology.

Yang Li received his B. Eng., M. Eng., and Dr. Eng. from the Southeast University, China in 1982, 1992 and 2002 respectively. Currently, he is a professor in Southeast University. His research interests include demand-side management and electricity market.

Yixin Ni (SM'94) received her B. Eng., M. Eng., and Dr. Eng. from Department of Electrical Engineering, Tsinghua University, China in 1968, 1981 and 1983 respectively. Her research interests are power system stability and control, HVDC transmission, FACTS, and electricity markets. She was a professor of Tsinghua University and is now with the University of Hong Kong. She is a senior member of CSEE and Fellow of HKIE. 